\title{
Changes in Spatial Scale in Drawing and Handwriting: Kinematic Contributions by Proximal and Distal Joints
}

\author{
F. Lacquaniti, ${ }^{1}$ G. Ferrigno, ${ }^{2}$ A. Pedotti, ${ }^{2}$ J. F. Soechting, ${ }^{3}$ and C. Terzuolo $0^{1,3}$ \\ ${ }^{1}$ Istituto di Fisiologia dei Centri Nervosi, CNR, Milan, Italy, ${ }^{2}$ Centro di Bioingegneria, Politecnico e Fond. Don Gnocchi, \\ Milan, Italy, and ${ }^{3}$ Laboratory of Neurophysiology, University of Minnesota, Minneapolis, Minnesota
}

\begin{abstract}
The coordination of arm and hand motions was studied in tasks involving drawing and handwriting movements of different amplitudes. To this end, pen motion was recorded by means of a digitizing table, while the positions of different markers on the limb were monitored by the ELITE system. It was found that the amplitude of shoulder and elbow angular motions scales roughly with the size of the figure drawn or of the script, whereas the amplitude of wrist and finger motions is small, independent of size. Consequently, the larger the limb movement, the smaller the magnitude of the contributions by motions at distal joints relative to that at proximal joints. Furthermore, while shoulder and elbow motions are tightly coupled (constant phase relation), motions at distal joints are loosely coupled to those at the proximal joints (variable phase relation). On the other hand, motion at distal joints increases the accuracy of the movement, as indicated by the smaller variability of pen trajectories compared to that of wrist trajectories.
\end{abstract}

It is well known that geometrical aspects, such as the shape, of a drawn figure or script can be preserved through wide changes in spatial scale (Lashley, 1930; Hebb, 1949; Bernstein, 1967). Also, the temporal profile of the velocity along the trajectory is independent of size (except for scaling in amplitude and time: Viviani and Terzuolo, 1980; Hollerbach, 1981), as is the relationship between velocity and curvalure (Lacquaniti et al., 1984). How such spatiotemporal invariances in the extrinsic space are mapped in the intrinsic space of the limb joint angles is a question that has not yet been addressed. In particular, one can ask what is the magnitude of the contributions to the movement by angular motions at proximal joints (shoulder and elbow) and at distal joints (wrist and fingers), and how do these contributions change as a function of the scale of execution. A priori, one might not expect that angular motions would scale in amplitude, since small movements appear to involve mostly wrist and finger motion, while large movements involve mostly shoulder and elbow motion.

We also aimed at describing the temporal aspects of the coordination of arm and hand motions during drawing and handwriting. It has previously been shown that fixed relations exist between shoulder and elbow instantaneous angular position in

Received Apr. 28, 1986; revised Sept. 8, 1986; accepted Sept. 10, 1986.

This work was supported by the Italian National Research Council, United States Public Health Service Grant NS-15018, and National Science Foundation Grant BNS-8418539.

Correspondence should be addressed to Dr. Francesco Lacquaniti, Istituto di Fisiologia dei Centri Nervosi, CNR, Via Mario Bianco 9, 20131 Milan, Italy. Copyright (C) 1987 Society for Neuroscience 0270-6474/87/030819-10\$02.00/0 both pointing (Soechting and Lacquaniti, 1981; Lacquaniti et al., 1986) and drawing (Soechting et al., 1986). However, in thesc studics motion was restricted to the proximal joints. As for angular motion at the wrist, it has been found to be largely uncoupled from shoulder and elbow motions during pointing and reaching (Lacquaniti and Soechting, 1982; Soechting, 1984). However, the degree of coupling could well be task dependent.

In order to address these points, we studied drawing and handwriting by combining 2 techniques designed for minimal interference with limb movement. Pen motion was recorded by means of a digitizing table, while the position of different markers on the limb was monitored by a recently developed optoelectronic system (ELITE: Ferrigno and Pedotti, 1985).

\section{Materials and Methods}

Motor tasks. Right-handed subjects were asked either to draw circles repetitively (5-10 cycles) or to write a single word on a digitizing table placed horizontally in front of them. The writing implement closely resembled an ordinary ballpoint pen, and the setup permitted a comfortable writing posture. The subjects were asked to vary the size of the circle or of the script. They freely produced movements within a square $60 \mathrm{~cm}$ to a side, the exact amplitude and location of the figure or script within this area being left unspecified ( 9 experiments with 4 subjects).

In 2 other experiments, the subjects were required to trace under visual guidance a word previously written by themselves and electrostatically copied at 7 different sizes, covering in geometrical progression (ratio $\sqrt{2}$ ) a range from 2.5 to $20 \mathrm{~cm}$.

Recording system. The $x, y$ coordinates of the movement described by the pen tip were recorded by means of an electromagnetic digitizing table (Calcomp 9240) at $100 \mathrm{~Hz}$ with a spatial resolution of $0.025 \mathrm{~mm}$, while the positions of different limb scgments werc recorded by the ELITE system. To this end, a $50 \mathrm{~Hz}$ TV camera monitored small reflective markers placed on the skin overlying bony landmarks at the shoulder, elbow, wrist, and the second metacarpophalangeal (MCP) joint (Fig. 1). The ELITE processor for shape recognition computes the $x, y$ coordinates of the marker centroids by performing in real time a bidimensional cross-correlation between the TV digitized image and a predetermined mask modeling the marker shape. Since the camera was positioned orthogonally above the writing table, the coordinates of the projection of the markers on the table were actually measured. The measured coordinates were scaled and corrected for optical distortion by means of a calibration procedure involving a $60 \times 60 \mathrm{~cm}$ grid with 121 equally spaced markers. The experimental values were calibrated using the parameters of a 2-dimensional power series mapping of the plane (into itself) that minimized the quadratic error between the measured and actual coordinates of the grid markers. With this method, the spatial accuracy of the system is about $0.3 \mathrm{~mm}$ (Ferrigno and Pedotti, 1985).

Data analysis. Angular orientation of the arm and hand on the plane was defined in terms of anatomical angles. The angles of horizontal rotation (yaw) at the shoulder $(\Theta)$, elbow $(\Phi)$, and wrist $(\Psi)$ are formed by the projection onto the horizontal plane of the upper arm, forearm, and metacarpus, respectively. They were computed trigonometrically from the $x, y$ coordinates of the limb markers. As a global estimate of the angular changes at the MCP and interphalangeal joints of the thumb 


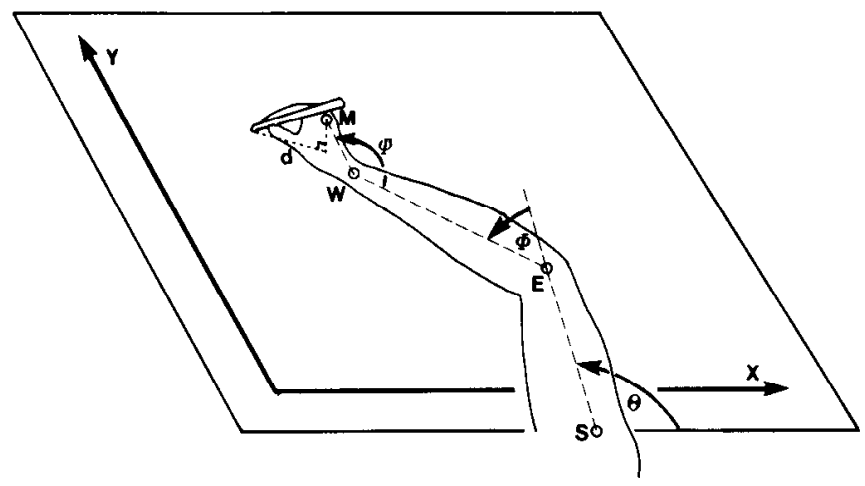

Figure 1. Schematic view of the experimental setup. Drawing and handwriting movements were execuled on a horizontal table. Pen motion was recorded by the digitizing table, while the positions of markers placed on the second metacarpophalangeal joint $(M)$, wrist $(W)$, elbow $(E)$, and shoulder $(S)$ were monitored by a TV camera placed above the table and were reconstructed by the ELITE processor. Yaw angles at the wrist $(\Psi)$, elbow $(\Phi)$, and shoulder $(\Theta)$ were computed trigonometrically from the marker coordinates. The projected distance $(d)$ between the pen tip and the marker on the metacarpal was also computed.

and index fingers (holding the pen), the projected distance $(d)$ between the pen tip and the marker on the second MCP joint was computed. Kinematic data were smoothed with a double-sided exponential filter (10 Hz cutoff). Their time derivatives were computed by numcrical differentiation ( 5 point Lagrange interpolation).

In the experiments in which subjects drew circles, the oscillatory changes in the $x$ - and $y$-components of the velocities at the pen and at the markers, as well as in the joint angular velocities, were (least squares) fitted with sinusoids in order to derive amplitude and phase of the fundamental (Soechting et al., 1986). Harmonic distortion was computed as the root-mean-square difference between the fundamental and the experimental value, normalized by the latter's deviance. Cycle-tocycle spatial variability of the $x-y$ trajectories described by the pen tip and by limb markers was quantified in 2 ways. (1) Each cycle of the movement was binned in 20 portions of equal temporal duration. In each bin, $95 \%$ confidence ellipsoids of $x, y$ coordinates were computed. As an overall estimate of the spatial variability, the areas of all ellipsoids were summed and normalized by the mean radius of the circle. (A similar procedure has been used by Georgopoulos et al., 1981.) (2) The average centroid of the $x-y$ trajectories was computed over all cycles. The mean coefficient of variation of the radial distance of each point on the trajectory from the centroid was then computed for 20 equal spatial sectors. Finally, the values of the coefficients were averaged over all sectors.

Script was analyzed in the following way. The $x-y$ trajectories at the pen and at the markers were rotated in the plane so as to align the major axis of the word with the $x$-axis. In order to quantify the differences between the trajectories described by the pen and those of the markers, the $x$ - and $y$-components of the velocity of MCP and wrist markers were scaled in amplitude so as to best fit (in the least-squares sense) the corresponding components of the pen velocity. A residual error was computed as the root-mean-square difference between the scaled velocities and the pen's velocities, normalized by the latter's deviance.

\section{Results}

\section{Circles}

The relative contributions to drawing movements by the angular motions at proximal and distal joints can be assessed by comparing the $x-y$ trajectories traced by the pen tip with those traced by the markers placed on the second MCP and on the wrist. Indeed, while the trajectory described by the wrist marker depends only on shoulder and elbow motions, MCP trajectory is also affected by wrist rotation and the pen's trajectory by flexionextension at the fingers. Figure 2 shows typical results from 1 subject drawing 2 circles of different sizes. (The diameter of the circle of Fig. $2 A$ was more than 6 times larger than that of Fig. 2B.)

In general, with medium to large circles, MCP trajectories roughly matched pen trajectories in terms of both shape and size, whereas wrist trajectories tended to be characteristically elliptical and slightly smaller. The discrepancy between the trajectory of the pen and the trajectories of the distal markers on the limb was much greater in the case of small circles, for which the difference in size between the 2 sets was an appreciable fraction of the size of the circle drawn by the pen. Furthermore, the trajectories described by MCP and wrist markers were consistently more distorted and had a larger cycle-to-cycle variability than the pen's trajectories. These results were independent of the relative location on the table where circles were drawn.

Previous work has shown that circular and elliptical figures are produced by the superposition of approximately harmonic oscillations in 2 orthogonal directions of the plane of motion (Hollerbach, 1981; Lacquaniti et al., 1983; Soechting et al., 1986). Left and right panels of Figure $2 C$ demonstrate such a result for the circles of Figure 2, $A$ and $B$, respectively. The thin traces correspond to the $x$ - and $y$-components of pen velocity, while the thick traces correspond to $\dot{x}$ and $\dot{y}$ of the MCP marker. Sine waves wcre fitted to these curves, and their amplitude, phase, and harmonic distortion computed. In all cases, for both pen and MCP trajectories, the amplitude of motion in the $x$ direction was about the same as that in the $y$ direction. (On average, the ratio between the larger amplitude and the smaller one was $1.09 \pm 0.07$ for pen trajectories and $1.14 \pm 0.21$ for MCP trajectories, $n=40$.) Furthermore, the phase difference between the $x$ - and $y$-components was $92 \pm 5^{\circ}$ at the pen and $91 \pm 16^{\circ}$ at $\mathrm{MCP}$.

Scatter diagrams of the data from 4 experiments (40 trials) are presented in Figure 3. The data points correspond to the ratios between the amplitude of MCP sinusoidal velocities and the amplitude of pen velocities (Fig. $3 A$ ) and to the corresponding ratios of the harmonic distortions (Fig. $3 B$ ). They are plotted versus the mean radius of the circle (ranging from about 0.5 to $18 \mathrm{~cm}$ ). Despite variability in the data, a clear trend is noticeable regarding the size of the circles. The ratio between the amplitudes of motion is a rapidly increasing and saturating function of the mean radius of the circle, while the ratio between the distortions is a rapidly decreasing function. Thus, while at the lower end of the range of circles the amplitude of motion at MCP was only about $50-60 \%$ of that of the pen, the proportion increased and leveled off at about $90 \%$ for circles with a radius of more than about $5 \mathrm{~cm}$. As for the harmonic distortion of the sinusoidal motion, it was generally low for pen trajectories (mean value across all experiments, including both $x$ and $y$ values: $3.6 \pm 2.7 \%$ ). The distortion of MCP (and wrist) trajectories was comparable to that at the pen for medium to large circles, but it was higher (about twice on the average) for circles less than $5 \mathrm{~cm}$ in radius.

A similar trend also characterized the relationship between the spatial variability of $\mathrm{MCP} x-y$ trajectories and the variability of pen trajectories. Cycle-to-cycle variability of the trajectories was computed in 2 different ways (see Materials and Methods): (1) as the total surface of $95 \%$ confidence ellipsoids of the $x, y$ coordinates of the mean trajectory and (2) as the average coefficient of variation of the radial distance of each point on the trajectories from the centroid. These 2 methods gave qualitatively similar results. Figure $3 C$ shows the results obtained with 

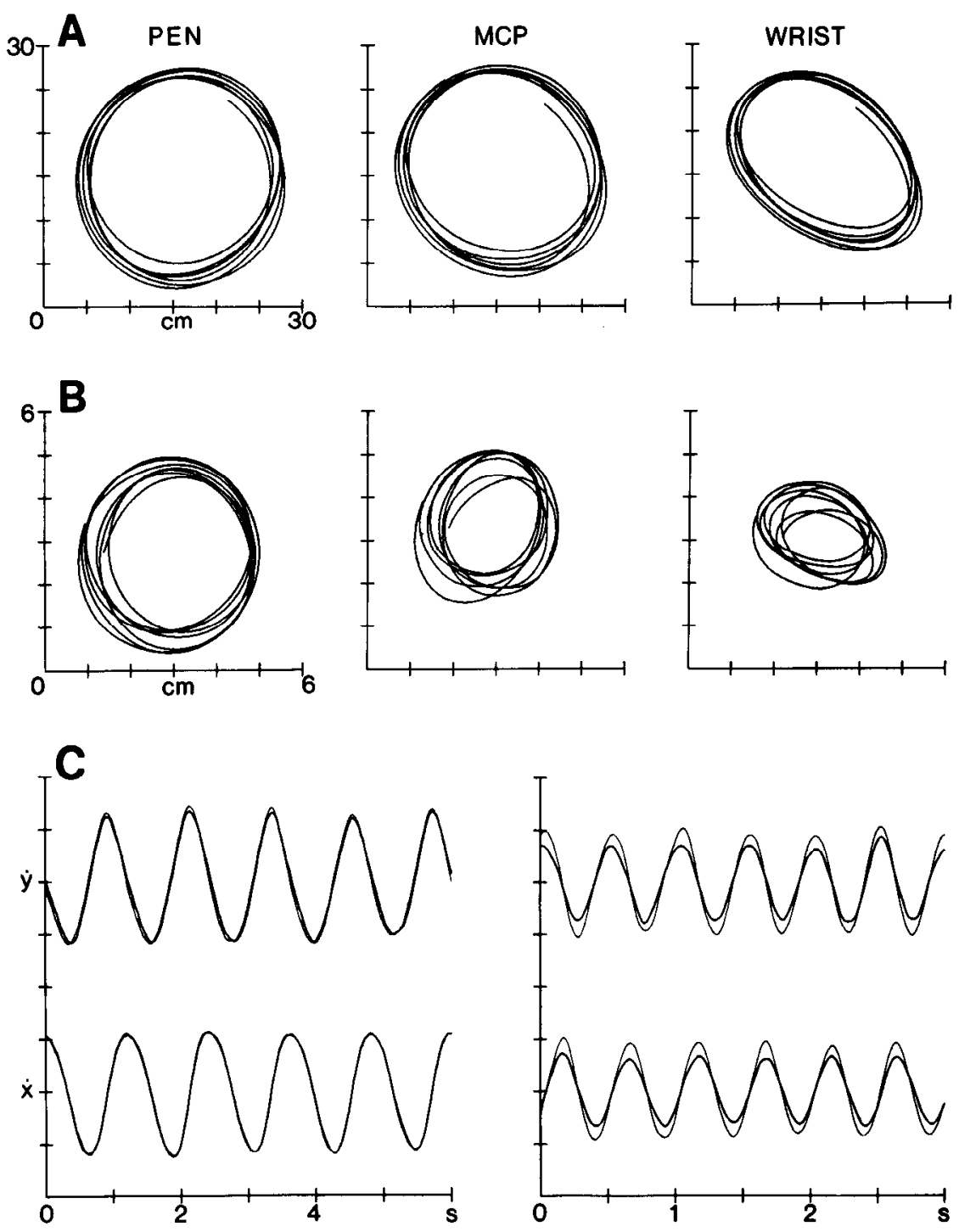

Figure 2. The $x-y$ trajectories for 2 circles of different size. $A$ and $B$, Trajectories at the pen tip, metacarpal marker, and wrist marker are plotted in the first, second, and third column, respectively. The mean radius of the circle traced by the pen is $11.2 \mathrm{~cm}$ in $A$, and $1.7 \mathrm{~cm}$ in $B$. C. The $x$ - and $y$-components of pen velocity (thin traces) and metacarpal velocities (thick traces) are plotted for the circle of $A$ in the left panel, and for that of $B$ in the right. Scales (per division), 50 (left) and 25 $\mathrm{cm} / \mathrm{sec}$ (right).

the second method. Each data point corresponds to the ratio between the average coefficient of variation of MCP trajectories and the corresponding coefficient of variation of pen trajectories. It should be noted that, despite the absence of experimental constraints on the accuracy of execution, cycle-to-cycle variability at the pen was rather limited (average coefficient of variation of pen trajectories, $8.3 \pm 4.5 \%$ ). The variability of MCP trajectories was close to that of pen trajectories in large circles, but it tended to increase with decreasing size of circles. At the lower end of the range, it was about twice the variability at the pen.

For circles and ellipses drawn by the arm in free space, harmonic oscillations in 2 orthogonal directions of the plane of motion result from sinusoidal changes in orientation angles of the shoulder and elbow joints with fixed phase relations (Soechting et al., 1986). Here, we shall show that the motion at distal joints is also oscillatory. However, while the amplitude of the angular motion at the shoulder and elbow joints scales roughly with the radius of the circle, the amplitude of angular motion at the wrist joint and of linear motion at the finger tips remains small, independent of the size of the circle. Figure $4 A$ illustrates this point with the data from the same trial as in Figure $2 A$, and Figure $4 B$ with those of Figure $2 B$. The top 3 traces in each panel are the temporal profiles of the angular velocity at the shoulder $(\dot{\Theta})$, elbow $(\dot{\Phi})$, and wrist $(\dot{\Psi})$ joints, while the trace at the bottom $(d)$ describes the changes in the time derivative of the projection on the drawing table of the distance between the pen tip and the marker at MCP joint. Changes in such distance provide an estimate of the combined angular motions at the MCP and interphalangeal joints of the thumb and index fingers.

It can be seen that the angular motions at the shoulder and elbow joints were approximately sinusoidal (the distortions ranged from 2 to $5 \%$ in the data of Fig. 4), the amplitude of elbow motion being slightly greater than that of shoulder motion (see Table 1). Note that the amplitude of shoulder and elbow motions in the case of the large circle (Fig. $4 A$ ) was about 10 times that in the case of the smaller one (Fig. $4 B$ ). (In Fig. $4 A$, the amplitude of $\Theta$ was $17^{\circ}$ and that of $\Phi$ was $23^{\circ}$; in Fig. $4 B$, the amplitudes of both $\Theta$ and $\Phi$ were $2^{\circ}$.) As for the phase relation between $\Theta$ and $\Phi$, it was invariably close to $140^{\circ}$ ( $\Phi$ lagging behind $\Theta)$, independent of the size of the circle.

Also the changes in wrist angle and in the projected distance between the pen and MCP were modulated in an oscillatory manner. However, their harmonic distortion was high, particularly in the case shown in Figure $4 A$ (where the distortion of $\Psi$ was $42 \%$ and that of $d$ was $52 \%$; in Fig. $4 B$, the distortions 

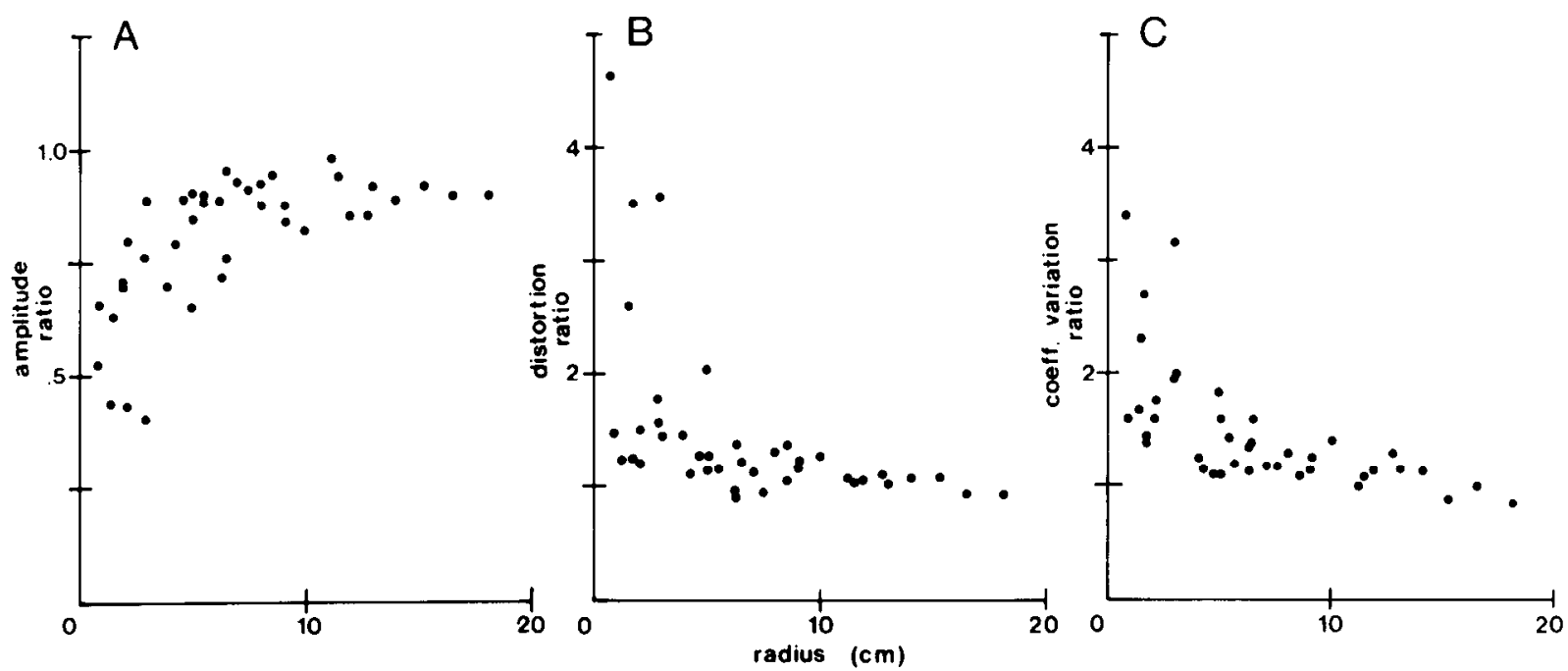

Figure 3. Scatter diagrams of the data from all drawing experiments. Each point represents the results of 1 trial $(n=40)$. In each panel, the abscissa values correspond to the mean radius of circles estimated as the amplitude of the sinusoid fitted to pen velocities (mean value of the fit to $\dot{x}$ and $y$ ) divided by the frequency of oscillation. $A$, Ratios between metacarpal and pen velocity amplitude. $B$, Corresponding ratios of the harmonic distortions. $C$, Ratios of the coefficients of variation of radial distances from the circle centroid.

were 11 and $15 \%$, respectively). ${ }^{4}$ Furthermore, despite the more than 6-fold difference between the radius of the circle of Figure $4 A$ and that of Figure $4 B$, the amplitude of finger and wrist motion did not differ appreciably in the 2 cases. (The amplitude of $\Psi$ was $1.6^{\circ}$ in $A$ and $1.3^{\circ}$ in $B$, while the amplitude of $d$ was $0.65 \mathrm{~cm}$ in $A$ and $0.53 \mathrm{~cm}$ in $B$.)

Figure 5 and Table 1 provide a summary of the data from all experiments and subjects. In Figure 5, the amplitude of angular motion at the shoulder $(A)$, elbow $(B)$, wrist $(C)$, and the projected distance between the pen and MCP $(D)$ are plotted versus the mean radius of circles. The angular changes in both $\theta$ and $\Phi$ scaled in amplitude with the radius of the circles through the whole range of movements examined. However, the ratio between the amplitude of shoulder and elbow motion and the radius of the circle was generally higher in large circles than in small circles. This is to be expected from the previous observation that the extent of the trajectory at the wrist, due to shoul-

${ }^{4}$ In general, the amount of distortion of $\Psi$ and $d$ was not correlated with the size of the circle. der and elbow motions, was a greater fraction of the trajectory at the pen in large circles than it was in small circles. A boundary between the 2 classes of circles was arbitrarily set at $5 \mathrm{~cm}$ of radius (see Fig. $3 A$ ), and a (least squares) regression line was fitted to the data points corresponding to circles of radius $>5$ $\mathrm{cm}$. The coefficient of correlation was 0.90 for the $\Theta$ angle and 0.97 for $\Phi$ angle.

No correlation existed, however, between the amplitude of wrist (Fig. 5C) or finger (Fig. 5D) motion and the radius of the circle. Indeed, the amplitude of motion at both $\Psi$ and $d$ was small, with little variation. (The apparent scatter of the data points results from the scales of the plots.) As can be seen in Table 1 , the average value of the amplitude of $\Psi\left(A_{\Psi}\right)$ was less than $3^{\circ}$ (with a SD $<1^{\circ}$ ), while the average amplitude of $d\left(A_{d}\right)$ was $<1 \mathrm{~cm}$ (SD $<1 \mathrm{~cm}$ ). The data in Table 1 also indicate that, as previously mentioned, the phase relation between $\Theta$ and $\Phi$ was close to $140^{\circ}$ with a small SD in all subjects, whereas the phase relations between $\Psi$ and $\Phi$ and between $d$ and $y$ (the component of pen motion in the direction of the changes of $d$ ) were highly variable, both among subjects and for the same
Figure 4. Time course of joint angular velocities and of the linear motion at the fingertips. The top 3 traces in each panel correspond to the angular velocity at the shoulder $(\dot{\Theta})$, elbow $(\dot{\Phi})$, and wrist $(\dot{\Psi})$. The bottom trace $(\dot{d})$ corrcsponds to the time derivative of the projected distance between the pen tip and the metacarpal marker. $A$, Data from the same trial as in Figure $2 A$. $B$, Data from Figure $2 B$. Scales (per division): $160(A)$ and $80 \% / \sec (B)$ for $\dot{\theta}, \dot{\Phi}$, $\dot{\Psi} ; 20(A)$ and $10 \mathrm{~cm} / \mathrm{sec}(B)$ for $d$.
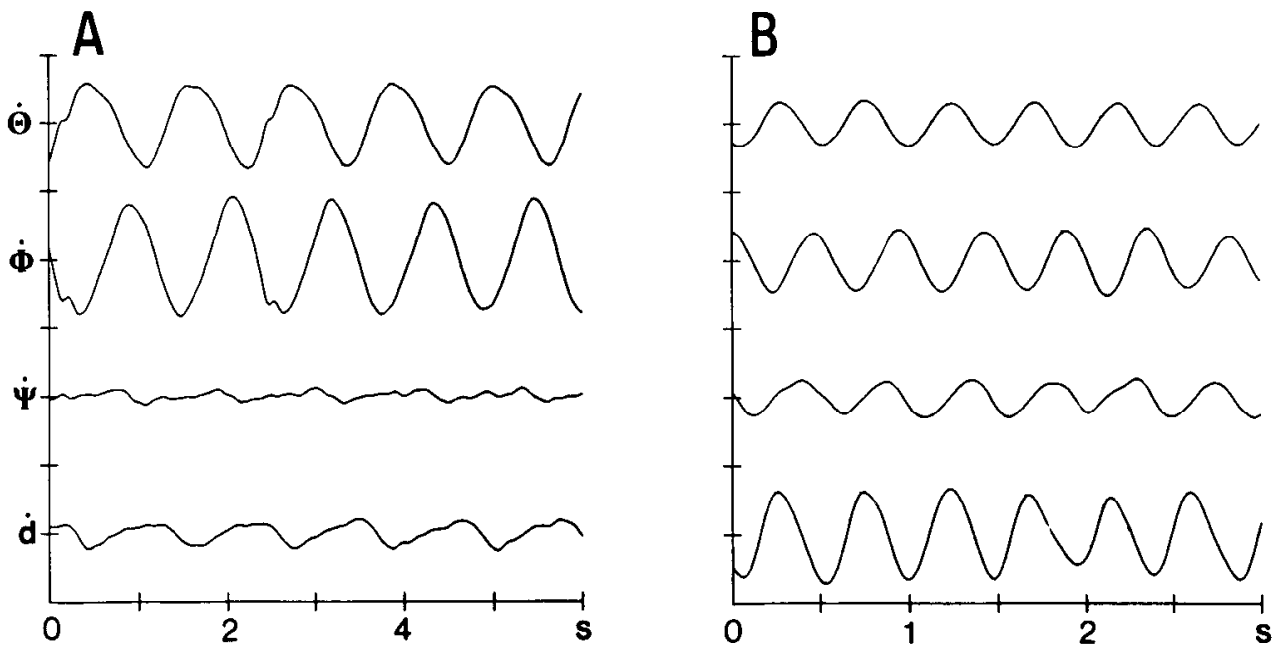
subject. The data of Table 2 demonstrate that these results were independent of the circle radius.

In summary, the amplitudes of shoulder and elbow motions scale roughly with the size of the circle, whereas the amplitudes of wrist and finger motion are independent of size. Furthermore, while shoulder and elbow motion are tightly coupled, motions at distal joints are loosely coupled to those at the proximal joints.

\section{Script}

We have shown that drawing movements involve a relative contribution by wrist and hand motion that depends on the size of the circle. The smaller the circle drawn, the greater the contribution by motion at distal joints relatively to that at proximal joints. Such a relationship with size was also demonstrated for handwriting. In these experiments, subjects were asked to write a single word in different sizes.

Figure 6 shows the typical results obtained in one such experiment. In Figure $6 A$ the word gatto (cat) was written on a large scale (overall horizontal extent, $22 \mathrm{~cm}$ ), while in Figure $6 B$ the same word was written in a much smaller size $(2.6 \mathrm{~cm})$. The top panels illustrate the $x-y$ trajectories at the pen tip, while the bottom panels illustrate the trajectories at the MCP marker. One can note that there is a close correspondence between MCP and pen trajectory only in Figurc $6 A$, but not in $6 B$.

However, when the $x$ - and $y$-components of pen motion are compared with those of the motion at MCP and wrist markers, similarities in the kinematics become apparent even in the case of small script. In Figure 7, the thin traces describe the $x$ - and $y$-components of pen velocity; the thick traces describe the velocities of the MCP (top panels) and wrist (bottom panels) markers, each velocity component having been scaled in amplitude to fit the corresponding component of pen velocity (see Materials and Methods). The data of Figure $6 A$ are plotted in Figure $7 A$; those of Figure $6 B$ in $7 B$.

In the case of the large word (Fig. 7A), the scaling factors for MCP velocities were 1.07 for $\dot{x}$ and 1.24 for $\dot{y}$; those for wrist velocities were 1.47 and 1.33 , respectively. After scaling, the correspondence between MCP and wrist velocities and the velocities at the pen was close; the residual error amounted to $13 \%$ for both $\dot{x}$ and $\dot{y}$ of MCP marker, and to 37 and $19 \%$ for $\dot{x}$ and $\dot{y}$, respectively, at the wrist. Some degree of wrist rotation amplified the extent of motion in the horizontal direction and modified the shape of the trajectory traced by shoulder and elbow joints.

In the case of the smaller script (Fig. $7 B$ ), the scaling factors for MCP velocities were 1.19 for $\dot{x}$ and 3.26 for $\dot{y}$; those for wrist velocities, 2.16 and 7.28 , respectively. The residual crrors were $56 \%(\dot{x})$ and $64 \%(\dot{y})$ at MCP, and $86 \%(\dot{x})$ and $76 \%(\dot{y})$ at the wrist. Although the fitting was generally poor with small script, similarities existed between the time course of MCP and wrist velocities and the time course of pen velocities. However, the degree of correspondence between the 2 sets of curves was not uniform within a single trajectory.

A summary of 3 experiments ( 50 trials) is presented in Figures 8 and 9 . They involve production of words with the same number of letters (5). ${ }^{5}$ The residual errors (Fig. 8) and the scaling factors (Fig. 9) computed for the velocity curves of each trial are plotted versus the logarithm of the length of the word. Word length ranged from about 1 to $60 \mathrm{~cm} .{ }^{6}$ The errors at both MCP

\footnotetext{
${ }^{5}$ Two other experiments involving longer words (9 letters) gave similar results.

"The height of such letters as "a," "e," and "o" ranged from about $0.2-8 \mathrm{~cm}$.
}
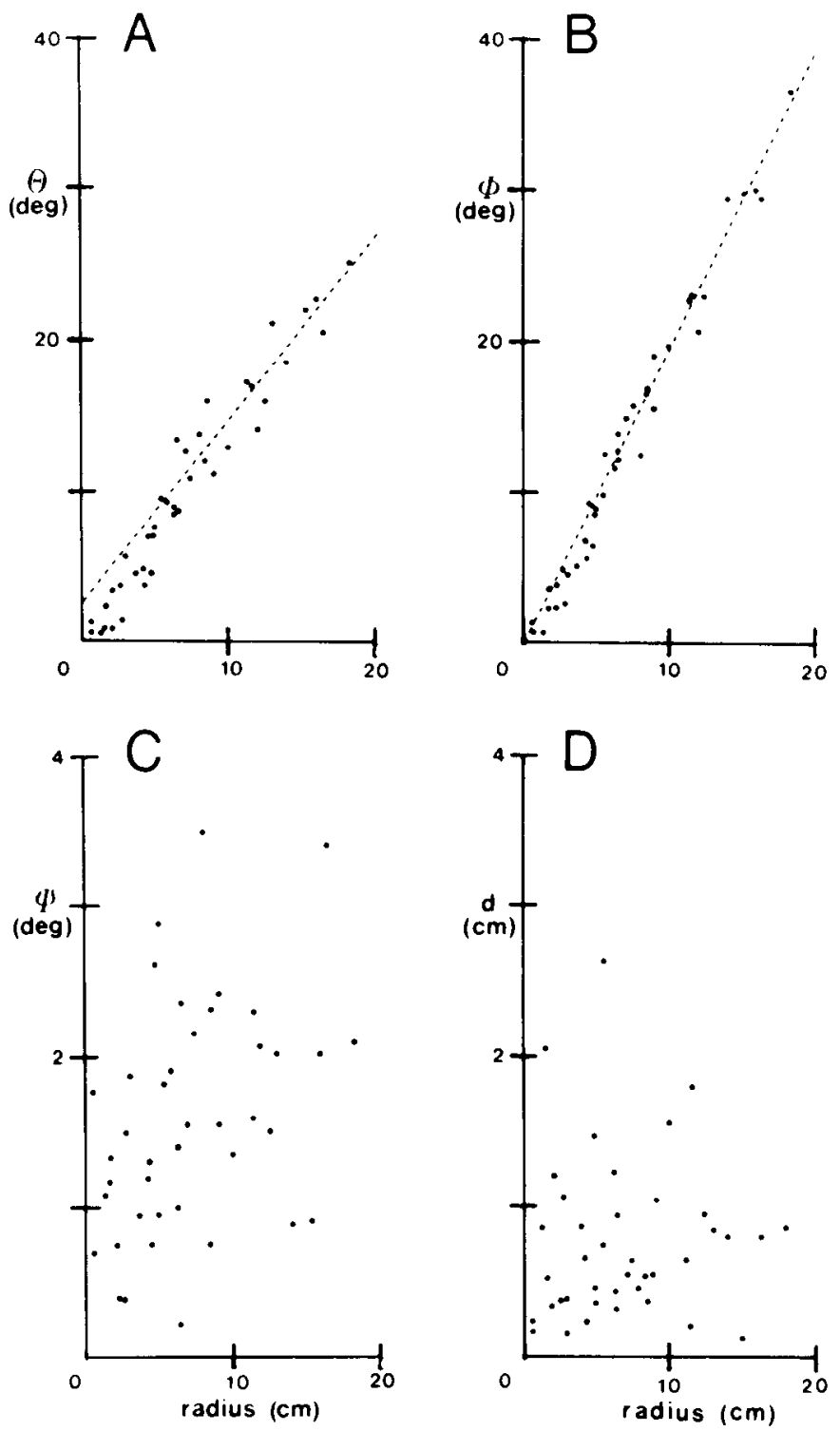

Figure 5. Amplitude of angular motions and finger motion as a function of circle radius. Amplitude of angular motion at the shoulder $(A)$, elbow $(B)$, wrist $(C)$, and projected distance between pen and metacarpal $(D)$. Data points are from the same trials as those of Figure 3. In $A$ and $B$ regression lines have been fitted to the points corresponding to circles of radius $>5 \mathrm{~cm}$. Their slopes are $1.20(A)$ and $1.97(B)$. Note that the amplitudes of $\Theta$ and $\Phi$ corresponding to circles of radius $<5 \mathrm{~cm}$ fall below the regression lines. No correlation exists between $\Psi$ and $d$ amplitude and circle radius. Note differences in scales.

and wrist were a rapidly decreasing function of the word length: they were generally less than 0.3 for words longer than about $10 \mathrm{~cm}$, but they were appreciably higher (often close to 1) for smaller words. Comparing the diagrams relative to MCP with those relative to the wrist, it can be noticed that the errors in the vertical component $(y)$ of wrist velocity were generally equivalent to the corresponding errors of MCP velocity. On the contrary, the errors in the horizontal component $(x)$ of wrist velocity were consistently greater than the corresponding errors of MCP velocity. This can be attributed to the fact that wrist rotation contributed mostly horizontal motion during handwriting.

The scaling coefficients of most trials involving words longer 
Figure 6. The $x-y$ trajectories for handwriting in different sizes. Pen and metacarpal trajectories are plotted in the top and bottom row, respectively. In $A$ the length of the word traced by the pen was $22 \mathrm{~cm}$; in $B, 2.6 \mathrm{~cm}$.
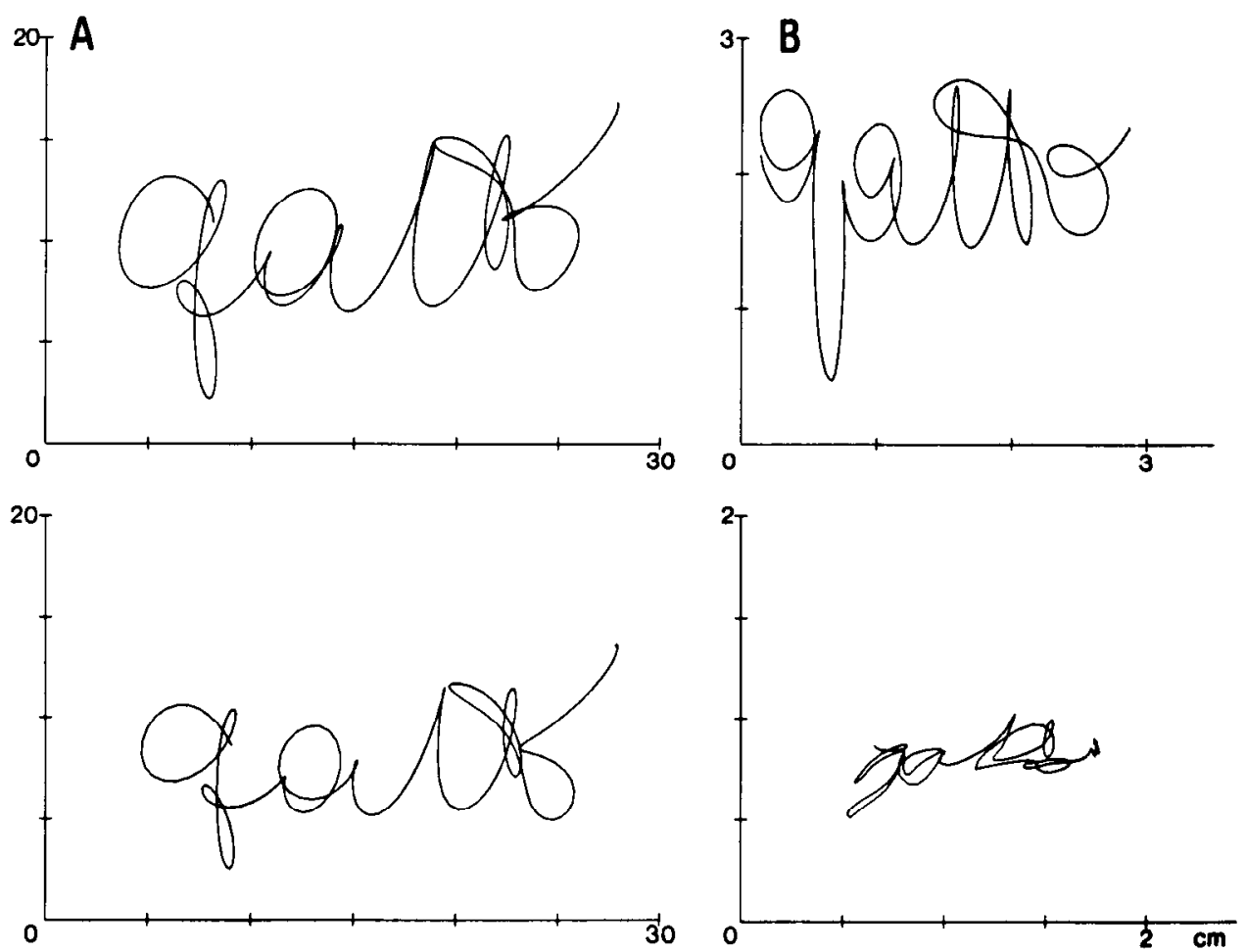

than about $10 \mathrm{~cm}$ clustered around 1-1.5 (Fig. 9). Indeed, as previously noted for large words, only a small difference existed in the extent of motion, in both the horizontal and the vertical directions, between the MCP or wrist markers and the pen. A much wider variability characterized, instead, the group of data involving small words. Generally, in such a group the scaling coefficients tended to be greater than 1 , in agreement with the observation that the trajectories described by MCP and wrist markers for small script were markedly shorter than the trajectory at the pen tip (see Fig. 6B). Some coefficients (particularly in the case of $x$ at the wrist) had either very small positive values or negative ones. It should be noted, however, that the presence of residual errors close to 1 in many such cases (Fig. 8) indicates the failure to reproduce pen trajectories with $\mathrm{MCP}$ or wrist trajectories by simply scaling the latter.
With regard to the changes in the joint angles during handwriting, the results were similar to those described for drawing: The amount of angular motion at the shoulder and elbow joints was roughly proportional to the size of the word, while the extent of angular motion at the wrist joint and of linear motion at the fingers was limited and independent of word length.

In 2 further experiments, subjects were asked to trace under visual guidance a given word previously written by them, and then reproduced in different sizes (see Materials and Methods). It can be simply stated that the results so obtained did not differ from those reported for free handwriting.

\section{Discussion}

Changes of spatial scale in drawing and handwriting on a horizontal plane involve nonuniform transformations of the con-

Table 1. Amplitude, phase, and distortion of joint angular motions and finger linear motion

\begin{tabular}{|c|c|c|c|c|c|c|c|}
\hline \multirow[b]{2}{*}{ Subject } & \multirow[b]{2}{*}{$n$} & \multirow[b]{2}{*}{$A_{\Phi} / A_{\Theta}$} & \multirow{2}{*}{$\begin{array}{l}A_{\Psi} \\
\text { (deg) }\end{array}$} & \multirow{2}{*}{$\begin{array}{l}A_{d} \\
(\mathrm{~cm})\end{array}$} & \multicolumn{2}{|c|}{ Phase difference (deg) } & \multirow[b]{2}{*}{$(d-y)$} \\
\hline & & & & & $(\Theta-\Phi)$ & $(\Psi-\Phi)$ & \\
\hline 1 & 10 & $1.27 \pm 0.20$ & $1.60 \pm 0.47$ & $0.53 \pm 0.17$ & $140 \pm 8$ & $87 \pm 14$ & $35 \pm 65$ \\
\hline 2 & 8 & $1.53 \pm 0.40$ & $1.11 \pm 0.86$ & $1.09 \pm 1.02$ & $137 \pm 11$ & $119 \pm 86$ & $98 \pm 67$ \\
\hline 3 & 18 & $1.37 \pm 0.12$ & $1.63 \pm 0.74$ & $0.88 \pm 0.43$ & $136 \pm 5$ & $33 \pm 22$ & $-85 \pm 117$ \\
\hline \multirow[t]{2}{*}{4} & 6 & $0.92 \pm 0.19$ & $2.52 \pm 0.61$ & $0.34 \pm 0.13$ & $142 \pm 11$ & $35 \pm 22$ & $-17 \pm 50$ \\
\hline & & \multicolumn{4}{|c|}{ Distortion (\%) } & & \\
\hline Subject & $n$ & $\bar{\theta}$ & $\Phi$ & $\Psi$ & $d$ & & \\
\hline 1 & 10 & $4.0 \pm 2.0$ & $3.7 \pm 1.8$ & $17.5 \pm 10.0$ & $36.0 \pm 24.0$ & & \\
\hline 2 & 8 & $5.9 \pm 6.3$ & $5.0 \pm 4.5$ & $59.8 \pm 27.4$ & $37.5 \pm 19.4$ & & \\
\hline 3 & 18 & $6.2 \pm 4.9$ & $4.6 \pm 4.2$ & $47.9 \pm 20.6$ & $40.3 \pm 19.8$ & & \\
\hline 4 & 6 & $4.2 \pm 4.4$ & $5.6 \pm 5.6$ & $16.8 \pm 8.9$ & $42.3 \pm 22.7$ & & \\
\hline
\end{tabular}



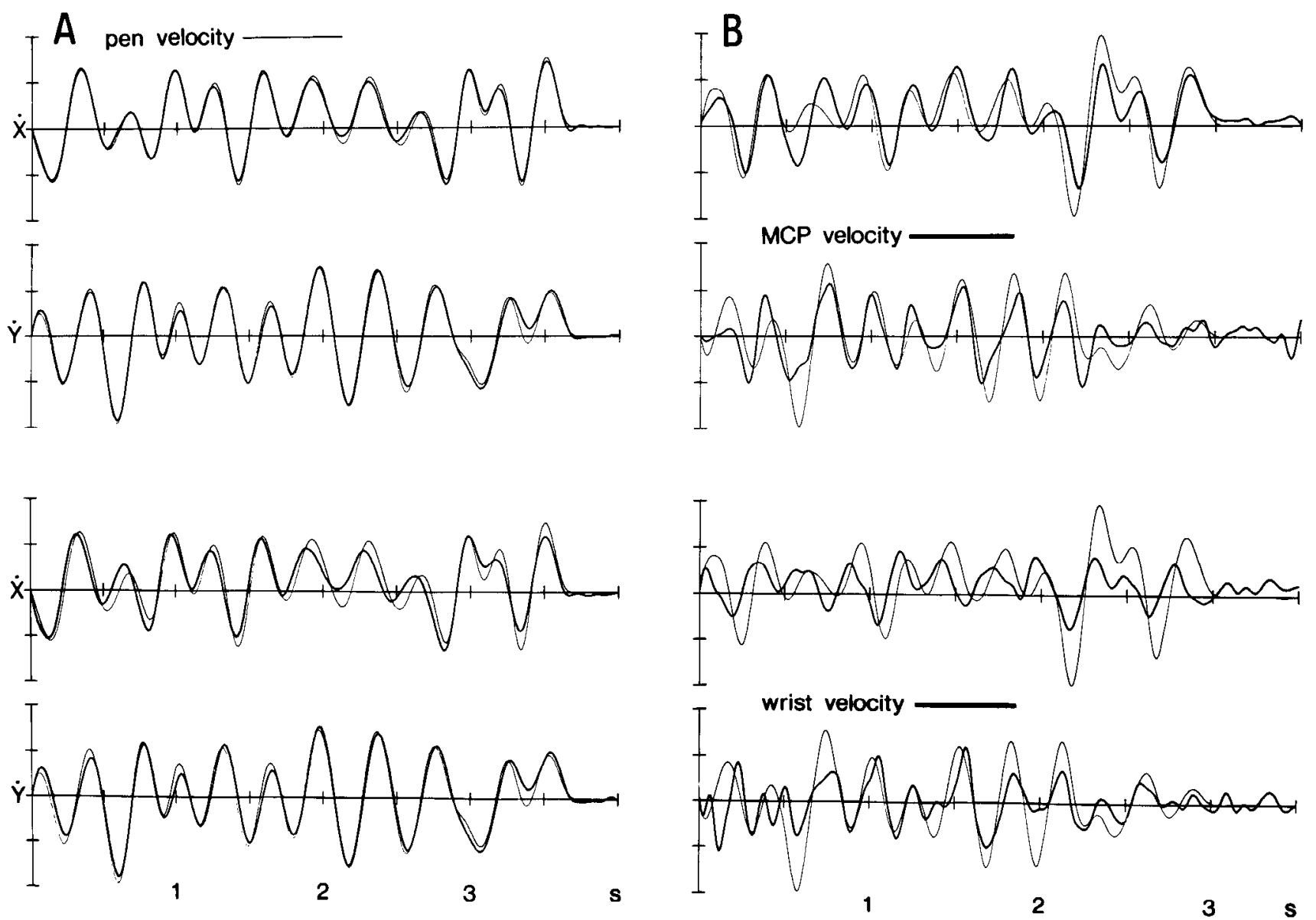

Figure 7. Time course of $x, y$ velocities for script. Thin traces correspond to the $x$ - and $y$-components of pen velocity; thick traces correspond to the velocities of metacarpal (top row) and wrist (bottom row), each velocity component having been scaled in amplitude to fit the corresponding component of pen velocity. $A$, Data from the same trial as in Figure $6 A$. B, Data from Figure $6 B$. Scales (per division): $50(A)$ and $6 \mathrm{~cm} / \mathrm{sec}$ $(B)$ for $\dot{x} ; 75(A)$ and $15 \mathrm{~cm} / \mathrm{sec}(B)$ for $y$.

tributing angular motions at the proximal and distal joints of the limb. While the amplitude of shoulder and elbow motions scales roughly with the size of the circle drawn or of the script, the amplitude of wrist and finger motions is independent of size. Consequently, the larger the circle or script, the smaller the contribution to limb movement by wrist and hand joint motion relative to that of the more proximal joints. This relationship is characteristically nonlinear, saturating rapidly with increasing size.

With regard to the absolute magnitude of the contribution by distal joints, the following should be noted. In general, the amplitude of the angular motion at the wrist and of the linear motion at the fingertips was surprisingly small. This cannot be attributed to biomechanical limits, since the possible range of movement at wrist and finger joints is far greater than what we observed (Andrews and Youm, 1979). Although it could be argued that the contribution by distal joints is overlooked in the present analysis, since the number of degrees of freedom at the wrist and hand is higher than that accounted for by the variables measured, a comparison of the $x-y$ trajectories described by the wrist with those described by the pen tip indicates that the global extent of motion contributed by the distal joints is indeed limited.

A second distinguishing element between motion at proximal joints and at distal joints is represented by the relative degree of dynamic coupling: shoulder and elbow motions are tightly coupled, whereas motions at distal joints are loosely coupled to those at the proximal joints (see Tables 1 and 2). Previous results concerning drawing movements of the arm in free space indicated the existence of fixed phase relations betwecn the oscil-

Table 2. Size independence of selected motion parameters

\begin{tabular}{llllllll}
$\begin{array}{l}\text { Radius } \\
(\mathrm{cm})\end{array}$ & $n$ & $A_{\Phi} / A_{\theta}$ & $\begin{array}{l}A_{\Psi} \\
(\mathrm{deg})\end{array}$ & $\begin{array}{l}A_{d} \\
(\mathrm{~cm})\end{array}$ & \multicolumn{3}{c}{ Phase difference $(\mathrm{deg})$} \\
\hline $0.0-6.9$ & 6 & $1.28 \pm 0.14$ & $1.36 \pm 0.63$ & $0.92 \pm 0.32$ & $132 \pm 7$ & $18 \pm 13$ & $-90 \pm 98$ \\
$7.0-13.9$ & 6 & $1.43 \pm 0.07$ & $1.62 \pm 0.58$ & $1.07 \pm 0.52$ & $138 \pm 4$ & $46 \pm 15$ & $-90 \pm 131$ \\
$14.0-20.9$ & 6 & $1.42 \pm 0.08$ & $1.93 \pm 0.95$ & $0.64 \pm 0.35$ & $137 \pm 2$ & $34 \pm 28$ & $-74 \pm 156$
\end{tabular}

Data from subject 3 have been grouped according to the mean circle radius. 

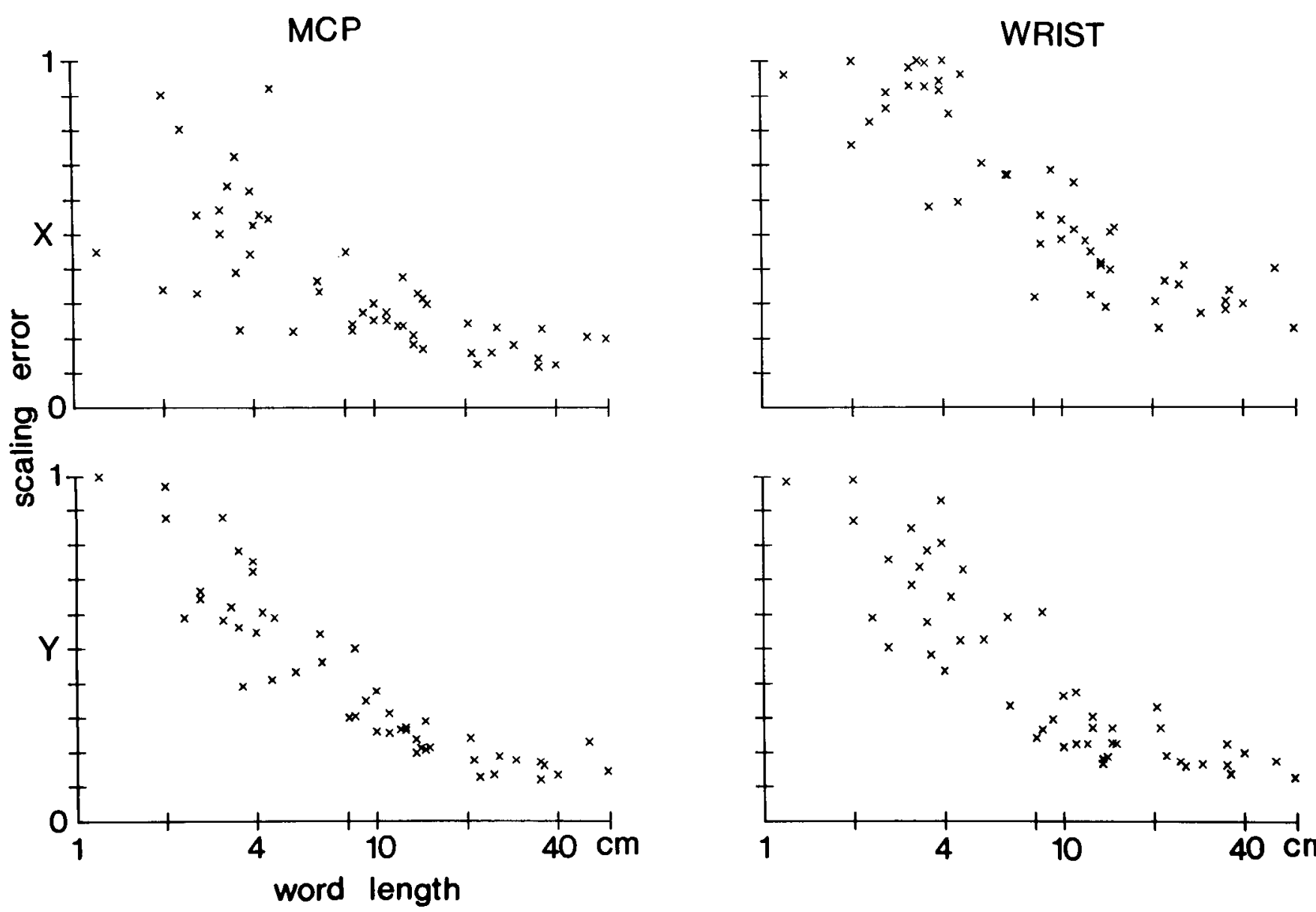

Figure 8. Scaling errors as a function of word length. Results from 3 experiments ( 50 trials). The residual errors of the scaling procedure for metacarpal (first column) and wrist (second column) velocities were computed as the root-mean-square difference between the scaled velocities and pen velocities, normalized by the latter's deviance. Values for $x$ in the top row; $y$ values in the bottom row.

latory changes in orientation angles of the shoulder and elbow (Suechting et al., 1986). However, in that study, motion was restricted to the proximal joints; furthermore, the effect of changes in scale of execution was not ascertained. The present results show that the changes in shoulder and elbow angles are constrained by a fixed phase relation even in the presence of concomitant motion at wrist and hand joints. Furthermore, the value of this phase relation is unaffected by changes in size of the drawn circle.

In contrast, angular motion at the wrist joint, as well as linear motion at the fingertips, exhibits a variable dynamic relationship with the concomitant motion at the proximal joints. From a mechanical viewpoint, angular motion at the wrist joint is inertially coupled to elbow and shoulder motions, in the sense that a torque applied solely at either the wrist or one of the proximal joints may result in angular motion at both joints. However, the moment of inertia of the hand is considerably less than that of the upper arm and forearm; consequently, torques at the wrist will have negligible effects on the motion at the more proximal joints. By contrast, elbow or shoulder torques do influence wrist motion, although this effect varies with the arm configuration. In fact, the degree of inertial coupling depends on the value of the angle formed by the hand and forearm in the plane of motion of the latter (Goldstein, 1980). Part of the variability among trials in the temporal relationship between wrist and elbow motions may therefore depend on variability of arm configuration. It is also to be expected that the proportion of wrist motion resulting from torque exerted at the proximal joints will be greater in larger movements of the limb than in smaller ones.

A variable degree of coupling between motion at the distal joints and motion at the proximal joints does not necessarily imply that the former are independent of the latter. On the contrary, we believe that the organization and control of limb movements by the CNS involves a tight coordination of the angular motions at proximal and distal joints. Several observations corroborate this. First, the extent to which both shape and size of wrist trajectories depart from the pen trajectories increases markedly with decreasing size of the circle or script, indicating that the contributions to the limb movement are variably coordinated and partitioned among proximal and distal joints according to the size of the movement. Furthermore, such partitioning may also vary within an individual trajectory, as indicated by the nonuniform degree of correspondence between wrist and pen velocities (see Fig. 7).

Also, when small circles are drawn, the spatial variability of the trajectories at the pen tip is reduced relative to that of the wrist trajectories. A somewhat similar result has been reported for pointing movements demanding a fair degree of accuracy (Soechting, 1984). In that case, the movement could be decomposed into 2 temporally distinct phases: a transport phase, in which the arm is brought toward the target with shoulder and 

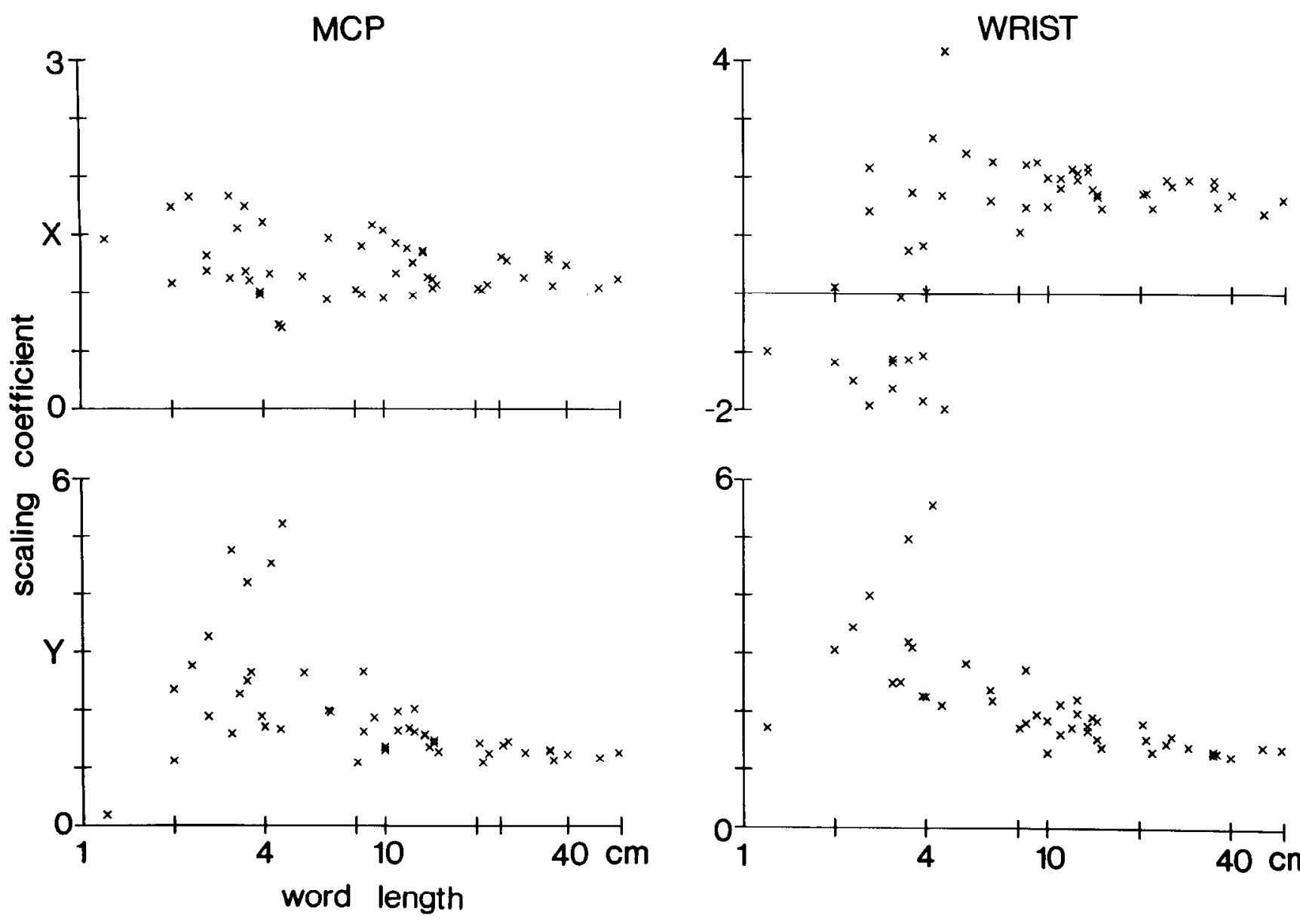

Figure 9. Scaling coefficients as a function of word length. Data are from the same trials as those in Figure 8.

elbow angular motion, and a "homing-in" phase, during which the hand is oriented relative to the target by means of a variable degree of wrist rotation. In proximity to the target, the spatial variability of finger trajectories was considerably less than that of wrist trajectories. Obviously, no such temporal decomposition can take place during drawing, and a higher degree of spatial accuracy is attained by the hand relative to the wrist throughout the movement. If motion at wrist and hand joints were independent of the motion at shoulder and elbow joints, inaccuracies of execution introduced by the latter would inevitably be reflected in the pen trajectory.

In conclusion, the present results point to the existence of two separate but coordinated domains of joint angular motion. For the tasks we examined, the proximal domain is characterized by constant phase relations between shoulder and elbow motions, while in the distal domain, joint motions have a small, roughly constant amplitude. One may hypothesize, therefore, that, at the level of movement planning, these constraints restrict the number of possible kinematic solutions (Greene, 1972). Within such boundaries, parameters of motion are specified based on the existing limb geometry (Lacquaniti et al., 1982) and the spatial and temporal scale of execution (Schmidt, 1982; Viviani and Terzuolo, 1983; Atkeson and Hollerbach, 1985; Flash and Hogan, 1985). Note that the solution for the proximal domain needs only to be approximate, since the trajectory described by shoulder and elbow motions seems to be taken into account in the implementation of motion at distal joints. Indeed, the increased accuracy of pen trajectories compared with that of wrist trajectories indicates the presence of corrective actions within the distal domain. One possibility that could account for our findings is that the virtual trajectory of the pen tip, such as would result from the superposition of a given set of motions at distal and proximal joints, is compared with the intended trajectory (as represented centrally), and any deviation gives rise to modifications of parameters of motion at distal joints. If so, efferent copy of the intended angular motions could be an appropriate input for determining the corrective actions (feedforward mode), but the participation of feedback mechanisms during the execution of the movement cannot be ruled out.

\section{References}

Andrews, J. G., and Y. Youm (1979) A biomechanical investigation of wrist kinematics. J. Biomech. 12: 83-93.

Atkeson, C. G., and J. M. Hollerbach (1985) Kinematic features of unrestrained vertical arm movements. J. Neurosci. 5: 2318-2330.

Bernstein, N. (1967) The Co-ordination and Regulation of Movements, Pergamon, Oxford, U.K

Ferrigno, G., and A. Pedotti (1985) ELITE: A digital dedicated hardware system for movement analysis via real-time TV signal processing. IEEE Trans. Biomed. Eng. $B M E-32$ : 943-950.

Flash, T., and N. Hogan (1985) The coordination of arm movements: An experimentally confirmed model. J. Neurosci. 5: 1688-1703.

Georgopoulos, A. P., J. F. Kalaska, and J. T. Massey (1981) Spatial trajectories and reaction times of aimed movements: Effects of practice, uncertainty and change in target location. J. Neurophysiol. 46: $725-743$. 
Goldstein, H. (1980) Classical Mechanics, Addison-Wesley, Reading, MA.

Greene, P. H. (1972) Problems of organization of motor systems. Prog. Theor. Biol. 2: 304-338.

Hebb, D. O. (1949) The Organization of Behavior. A Neuropsychological Theory, Wiley, New York.

Hollerbach, J. M. (1981) An oscillation theory of handwriting. Biol. Cybernet. 39: 139-156.

Lacquaniti, F., and J. F. Soechting (1982) Coordination of arm and wrist motion during a reaching task. J. Neurosci. 2: 399-408.

Lacquaniti, F., J. F. Soechting, and C. Terzuolo (1982) Some factors pertinent to the organization and control of arm movements. Brain Res. 252: 394-397.

Lacquaniti, F., C. Terzuolo, and P. Viviani (1983) The law relating the kinematic and figural aspects of drawing movements. Acta Psychol. 54: 115-130.

Lacquaniti, F., C. Terzuolo, and P. Viviani (1984) Global metric properties and preparatory processes in drawing movements. In Preparatory States and Processes, S. Kornblum and J. Requin, eds., pp. 357-370, Erlbaum, Hillsdale, NJ.

Lacquaniti; F., J. F. Soechting, and C. Terzuolo (1986) Path constraints on point-to-point arm movements in three-dimensional space. Neuroscience 17: 313-324.

Lashley, K. S. (1930) Basic neural mechanisms in behavior. Psychol. Rev. 37: 1-24.

Schmidt, R. A. (1982) Motor Control and Learning. A Behavioral Emphasis, Human Kinetics, Champaign, IL.

Soechting, J. F. (1984) Effect of size on spatial and temporal characteristics of a pointing movement in man. Exp. Brain Res. 54: 121132.

Soechting, J. F., and F. Lacquaniti (1981) Invariant characteristics of a pointing movement in man. J. Neurosci. 1: 710-720.

Soechting, J. F., F. Lacquaniti, and C. Terzuolo (1986) Coordination of arm movements in three-dimensional space. Sensorimotor mapping during drawing movement. Neuroscience 17: 295-311.

Viviani, P., and C. Terzuolo (1980) Space-time invariance in learned motor skills. In Tutorials in Motor Behavior, G. E. Stelmach and J. Requin, eds., pp. 525-533, North-Holland, Amsterdam.

Viviani, $P_{\text {., }}$ and C. Terzuolo (1983) The organization and control of movement in handwriting and typing. In Language Production, Vol. 2, B. Butterworth, ed., pp. 103-146, Academic, London. 